\title{
Hybrid Routing Topology Control for Node Energy Minimization for WSN
}

\author{
K Abdul Basith, T.N. Shankar \\ Department of Computer Science and Engineering \\ Koneru Lakshmaiah Education Foundation, Vaddeswaram, Guntur, Andhra Pradesh, India
}

\begin{abstract}
Wireless sensing has become an essential feature for minimizing energy for WSN applications. The foundation of the WSN is to implicate the uniqueness of the design feature capabilities, which are tied to different applications choices of interest. The implementation of pervasive algorithms with ubiquitous Network features are depicted with changes in frequency topology bands and congestion regression of the Network. The Network would affect the parametric criteria such as bitrate, Cluster-head energy, minimum energy and bandwidth usage. Our improved hybrid Pervasive algorithms would prevent the different attacks and control with the least tolerant error since topology becomes an integral part of the design, providing efficient Routing for the Network. In order to effectively solve the problem, a hybrid tangential transform with improved topologies for effecting network parameters. The other algorithm implicates the energy-efficient with optimization of stochastic conditional inequity for different network sizes. Performance characteristics of the proposed algorithms for WSN would estimate a tolerant error with a factor of $12 \%$ on each feature of the network parameter.
\end{abstract}

Keywords-SCI (stochastic conditional inequality); LEACH; clustering; DDOS (distributed denial of service); DEEC (distributed energy efficient clustering); TETRA (terrestrial trunked radio); WSN (wireless sensor network)

\section{INTRODUCTION}

The Wireless Sensor Network (WSN) affects everyday life as a seed for intelligent applications and ubiquitous systems. WSNs employ traditional WiFi antennas and a collection of internet-connected devices known as "smart nodes" capable of detecting and recording environmental phenomena and physical conditions such as humidity, temperature, pressure, and pollution levels.

Because of the technology's flexibility and communication capabilities, it is possible to exploit the accurate information generated by agent devices. Consequently, the communication is reliable because [1] and [2]. To ensure the long-term survival of innovative applications like IoT (Internet of Things) and military applications, WSNs have been used to demonstrate various intriguing ways. As shown in Fig 1, many smart apps rely on WSNs as their basis. The advent of different scenarios of WSN, which might include the different nodes and that will be deployed with dynamic nodes on each MANETS [3] where ADHOC features are improvised on multi-dimensionality feature [4]. Wireless sensor nodes and a data centre or sink node are the two sensors in this system [5]. It is these nodes that introduce three critical operations into the system.
- Data collection,

- Data processing, and

- Data transmission.

A sensor unit, a processing unit, a data storage unit, a radio transceiver unit, an energy unit, and a power generator are also included as functional modules for controlling and monitoring them. WSN incorporates the sending and reception of data from the data center or sinks node via a wireless channel [6, 9]. With the rise of countries with less established infrastructure, investment in wireless sensor networks (WSN) has become an inescapable consequence because of their low cost and high communication capabilities [10-13]. There are still substantial challenges in WSN related to network capacity restrictions, increasing data loss and collision rates [7]. By offering an efficient routing protocol based on clustering, it is possible to fulfil the Quality of Service (QoS) criteria while at the same time enhancing all-around network performance [8]. Recent years have seen an increased interest in WSN because of its adaptability and communication capabilities, particularly in the absence of conventional networks like Long-Term Evolution (LTE) or Terrestrial Trunked Radio (TETRA), both in academic research and in the IT sector [14-17]. As a result of these concerns and obstacles, the WSN's potential performance may be severely affected by factors such as frequent network topology changes and longer delays in reaching the final destination, route coupling and high packet loss. In order to lower network bandwidth and power consumption while simultaneously extending the lifespan of the Network, new approaches to congestion reduction are needed [18]. Also mentioned in this part are several popular strategies for dealing with congestion control issues and an overview of the topic in network layer congestion management [20].

\section{A. Contribution}

This study's primary objective implicates the different scenarios of attacks and its control feature using the pervasive algorithm for improving the overall performance features as throughput, end-end delay time, and the energy minimization for the proposed algorithms. This paper mainly contributes with implementation of routing cluster analysis with proposed route structures from III.B as stated below:

\section{B. Problem Statement}

1) Implement the Hybrid Routing protocol for minimum cluster head energy. 
2) Improvise a design model on each Routing area calculations for minimum distance measurement.

3) Estimate a stochastic model, for optimizing the error and formulate the relative error features on each node based on SCI algorithm.

4) Our proposed model, with DEEC-SCI and HTTA models have improvised to implicate different performance factors such as bit rate, energy minimization at nodes, and error optimization in dB's.

5) Finally, comparison of LEACH algorithms, TEEN algorithm, and DEEC \{SCI-HTTA\} (proposed algorithms) have been implicated as Tabulated graphs.

\section{Overview}

In Section-1, our design implicates different MANET's and their importance of congestion problem and its related features that are governed with Route optimization and region cluster analysis. Network topologies are estimated with different diagram feature consideration and their literature survey of different algorithms with routing protocols list. In section 3, we ensure the different possibilities of optimization algorithms that are governed for ensuring optimal solutions. Finally, we introduce the SCI-HTTA algorithm for energy minimization formulations.

\section{EXISTING DESIGN}

\section{A. Concept}

Congestion-aware clustering and Routing (CCR) is the purpose of this architecture is to improve network performance by lowering end-to-end delay time, boosting delivery ratio, and prolonging the network lifespan. As a result, several obstacles and concerns must be addressed to fulfil these goals, including the dependency on batteries, the capacity of storage units, and the need to send data to a specific receiver node many times [21].

- Low Overhead: Because the setup phase is done just once in the first round, the overhead associated with executing the setup step in each subsequent round is minimized. It is used during the setup phase to split the network area into levels and sectors, which are subsequently utilized to build clusters of nodes with an equal number of nodes once they have been created.

- Cluster head node $(\mathrm{CH})$ load distribution: As the functions of principal cluster head $(\mathrm{PCH})$ and secondary cluster head $(\mathrm{SCH})$ rotate among all nodes in the Cluster at the start of each cycle, the cluster head node $(\mathrm{CH})$ role is distributed over all nodes, hence reducing the strain on all nodes.

- Stability is achieved when data transfer with $\mathrm{SCH}-\mathrm{PCH}$ forms Cluster heads correctly.

- Reliability: The values of $\mathrm{PCH}$ and $\mathrm{SCH}$ should be more reliable and dependable for optimal distance calculations.

- Scalability refers to adding new nodes at any point during a round.
- Fault-tolerance: increasing the packet delivery ratio through the use of fault tolerance solutions is known as fault tolerance.

\section{B. Congestion Problem}

When a large number of sensor nodes submit data to a single sink node, there is a high likelihood of network congestion. Several factors contribute to this, including a relatively restricted bandwidth supply and finite network capacity [6]. Figure 2 is an illustration of this phenomenon.

For congestion to arise in a WSN, there are two primary reasons for this: a lack of node capacity and the characteristics of the wireless channel. Due to the prominent restricted resources, sluggish processor, and restricted energy of nodes, congestion in WSN occurs in the first place in the Network's nodes. Secondly, network congestion occurs in WSN because of the nature of the Network, its event-driven nature, channel interference, and the pace at which data is sent and received from the Network. Consequently, protocols developed for WSNs must be lightweight and scalable to maximize the Network's lifespan [22].

\section{Cluster-Based Route Optimization Protocols with Block- Based Cluster Formation}

The Low-Energy Adaptive Clustering Hierarchy Protocol (LEACH) [14] is a self-organizing, adaptive clustering protocol that uses little energy to perform its tasks. LEACH's procedure is divided into several rounds that are repeated repeatedly. Nodes form themselves into clusters at the end of each round. Each Cluster consists of a single $\mathrm{CH}$ node and a large number of MNs nodes, where the $\mathrm{CH}$ node receives data from the MNs nodes and executes data tasks simultaneously on the data before sending the aggregated data to the base station. It had several advantages, including balancing energy usage, using TDMA on the MAC, aggregating data from $\mathrm{CH}$ nodes, which resulted in a reduction in the high volume of traffic and savings in energy. In addition, it can create new nodes and eliminate dead nodes in each cycle. Nevertheless, it has some disadvantages, such as random selection of $\mathrm{CHs}$, residual energy that is not considered when selecting a $\mathrm{CH}$, single-hop inter-cluster Routing, which increases energy consumption in large-regional networks, and dynamic Cluster which adds extra overhead to the overall network design. Fig 2 is a collection of the most recent successors to the LEACH protocol, together with information on its approach, advantages, and disadvantages.

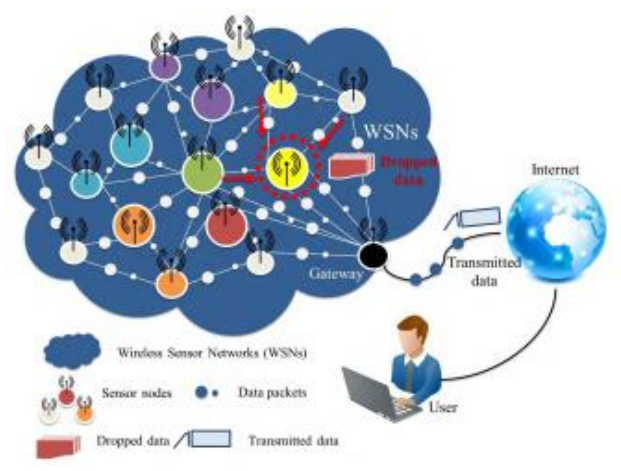

Fig. 1. Representing the Congestion Problem for WSN Systems. 


\begin{tabular}{|c|c|c|c|c|}
\hline SNO & ALGORITHMS & TECHNIQUE & ADVANTAGES & RESEARCH GAP \\
\hline 1 & LEACH-C [14] & $\begin{array}{l}\text { Implicate a cental } \\
\text { algorithm to form } \\
\text { clusters }\end{array}$ & $\begin{array}{l}\text { Improved } \\
\text { Performance by } \\
20 \% \text { to } 40 \%\end{array}$ & $\begin{array}{c}\text { Every Node requires } \\
\text { enables positional } \\
\text { analysis from GPS, } \\
\text { ence increases extra } \\
\text { energy }\end{array}$ \\
\hline 2 & LEACH-F[15] & $\begin{array}{c}\text { This design requires a } \\
\text { two level of } \\
\text { transmission. }\end{array}$ & No setup overhead & $\begin{array}{c}\text { Consumes much power } \\
\text { where new nodes and } \\
\text { impossible to remove } \\
\text { dead nodes. }\end{array}$ \\
\hline 3 & LEACH-E[16] & $\begin{array}{l}\text { The design on Cluster } \\
\text { head and its selections } \\
\text { implicates with amount } \\
\text { of resudal energy. }\end{array}$ & $\begin{array}{c}\text { Increased the } \\
\text { network lifetime by } \\
40 \% .\end{array}$ & $\begin{array}{l}\text { Fixed time round leads } \\
\text { to the waste of energy }\end{array}$ \\
\hline 4 & LEACH-ET[17] & $\begin{array}{l}\text { The design for this leach } \\
\text { model utilzies threshold } \\
\text { to increase the } \\
\text { proportion stedy state in } \\
\text { each round. }\end{array}$ & $\begin{array}{c}\text { Energy efficieny } \\
\text { enhanced }\end{array}$ & $\begin{array}{l}\text { This model consumes } \\
\text { more energy in } \\
\text { transmitting continous } \\
\text { message. }\end{array}$ \\
\hline 5 & LEACH-MH[18] & $\begin{array}{l}\text { Provides inter and intra } \\
\text { multi hop } \\
\text { communication model } \\
\text { to send and recive data } \\
\text { from the devices } \\
\text { implanted. }\end{array}$ & $\begin{array}{l}\text { Improvise a large } \\
\text { section of energy } \\
\text { reduction for large } \\
\text { size of network. }\end{array}$ & $\begin{array}{l}\text { Suffers from hot spots } \\
\text { and limited scalability }\end{array}$ \\
\hline
\end{tabular}

Fig. 2. Representing the Tabulated figure for List of Algorithms for Survey Model.

\section{Methodology}

With consideration of different design models on the design, LEACH models and its different types have been implicated with Fig-2. According to the current design which effectively improvise on congestion problem and Route areabased design features have been implicated on figure 3 and figure 4. Now, these LEACH models effectively improvise the Routing concept on the basis with region model and its collective intra-cluster routing algorithm mentioned in section F. The current design effectively postulates on different distance formulation with area and its effective scheduling. This feature estimation is represented with on algorithm 2 .

In [19], LEACH protocol with fixed number of Cluster head with each round based on random time interval. The PLEACH is proposed with optimal cluster-based chain protocol which implicates the improvement of PEGASIS and LEACH protocols as hybrid model for optimizing energy. An NS2 feature implementation of the WSN model with P-LEACH is performed and its energy feature reduction is affected on each set of optimal nodes considered [26]. In [27], the authors suggest a hybrid multihop routing protocol that attempts to increase the lifespan of a WSN that is deployed in a globally spread network while maintaining its performance. Despite the fact that its performance has been shown to be superior, the chain-based $\mathrm{CH}$ selection and routing of data over MST increases the network's energy consumption, hence shortening its lifespan. This concept creates a hybridization of the metaheuristic cluster-based routing (HMBCR) approach for use in wireless sensor networks. The HMBCR approach begins with a brainstorm optimization with levy distribution (BSO-LD) based clustering procedure that incorporates a fitness function including a fitness function comprising [28].

The event data flow in IMS applications must be delivered in a timely and reliable manner in order for the applications to respond quickly with the relevant actions. However, because of a sensor node's limited energy supply, it is necessary to make a trade-off between latency and energy consumption while selecting the best path to the base station. With the advent of event data traffic in IMS, a multi-objective antcolony optimization-based quality of service (QoS) aware cross-layer routing (MACO-QCR) protocol has been proposed for inter-cluster communication in WSN-based IMS in order to deal with the multi-constrained routing problem introduced by event data traffic. An improvement to the ACO algorithm is that it is now a multi-objective routing algorithm that considers the energy consumption cost and the end-to-end delay cost of a routing path as two optimization objectives, and in which a routing path is produced through the use of multi-pheromone information and multi-heuristic information that is comprised of two objective functions is used to generate the routing path [29].

In this design, the network itself is optimized for energy efficiency and routing endurance without taking into account the influence of the external environment, resulting in a network that is unable to adapt to environmental changes in a timely way. Thus, the routing survival of various routing protocols in severe conditions is a matter of some debate.

The SMRP (sustained multipath routing protocol), in which routing choices are determined based on a mixed potential field in terms of depth, residual energy, and environment To summarize, the core concept of SMRP is that it instructs messages to pick pathways that make a compromise between delivery delay, energy balance, and routing survival, among other factors [30].

\section{E. Protocols for Region Cluster Analysis and Scheduling}

A vertical seam or line of terminals is defined as an Edge D2d Communication (LBDD) [20-25] system, which divides the deployment area into two halves by dividing it vertically. In this context, the nodes positioned on this strip or line are inline nodes, which means that they are located on the strip or line. Information is captured and kept on this line to be accessed later if necessary. It is necessary to convey data from sensors to the line, where it is maintained in the first node that comes into contact with the data. It is possible to send a data query to the line, which will then propagate across the line until it reaches the cluster nodes storing and retrieving data from the inline storage system, at which point the inquiry will be stopped. The data is delivered straight to the sink through the inline node in the next phase, and the multicast routing process is brought to a close. It is believed that each node is aware of its geographic location and the geographic boundaries of the Network. a. Additionally, in addition to the fact that it is relatively simple to identify and configure, LBDD has the benefits of being easily accessible by both sources and sink nodes and having a reasonably low overhead for completing the operations mentioned above. There are some drawbacks to LBDD, such as the fact that it still relies on a live broadcast for spreading metadata along a line and that the line must be wide enough to accommodate hot spots. As a result of this, especially for extensive systems, the flooding on the line will result in a rise in total energy. 

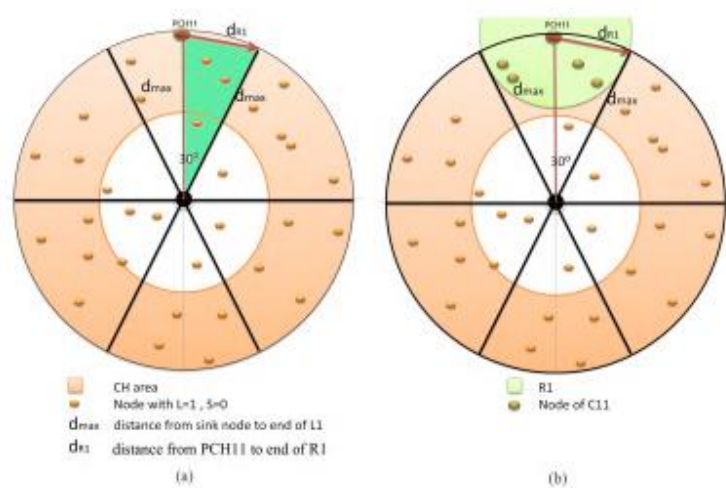

Fig. 3. Representing Area-based Protocol with the Optimum Route (a) Cluster Head Area with Minimum Distance for PCH, (b) Representing the Region of Cluster Nodes in R1 Region.

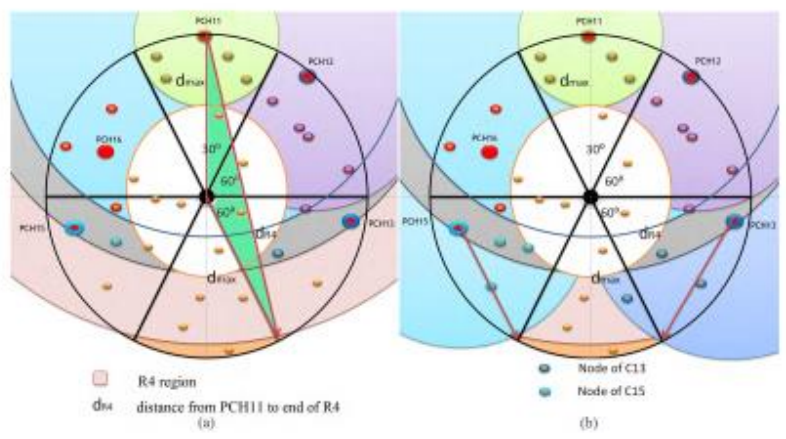

Fig. 4. Representing other Regions R4 with (a) Distance with PCH and other R4 (b) Node with Clusters with C11 and C15.

In Fig 3a\&4a, this design implicates each feature of improvements of region clusters with distance optimization on each section of segments with 30 degrees or any other angle $\emptyset$. Six sectors of the circular region are estimated with the different clusters and their distances D11 as calculated below.

$D_{11}=\sum_{i=1}^{N} D_{i} *\left(x_{i} \cos (\varnothing) \pm y_{i} \sin (\varnothing)\right)$

Similarly, Fig $3 b-4 b$ considers the distances from clusters and within subdivision region of the Network considered. Hence, the final distance is optimized as:

$D_{x}=\sqrt{(D 11 \cos (\rho)+D 11 \sin (\rho)}$

\section{Setup Phase}

\section{F. Setup Phase}

A single instance of the setup phase is performed in the first round, during which fixed clusters are arranged. In order to start the data transception of WSN, a list of clusters are generated with the capacity considered. Following this phase, the network area is subdivided into levels and sectors. A cluster is formed when each level and sector intersect with one another. It is also necessary for each node to know its cluster number, which is composed of a level number $\mathrm{L}$ followed by a sector number S, indicated by the letters Cls. Each Cluster must contain a PCH node and an optional $\mathrm{SCH}$ node. The $\mathrm{PCH}$ and $\mathrm{SCH}$ nodes are picked during the setup phase based on their distance.

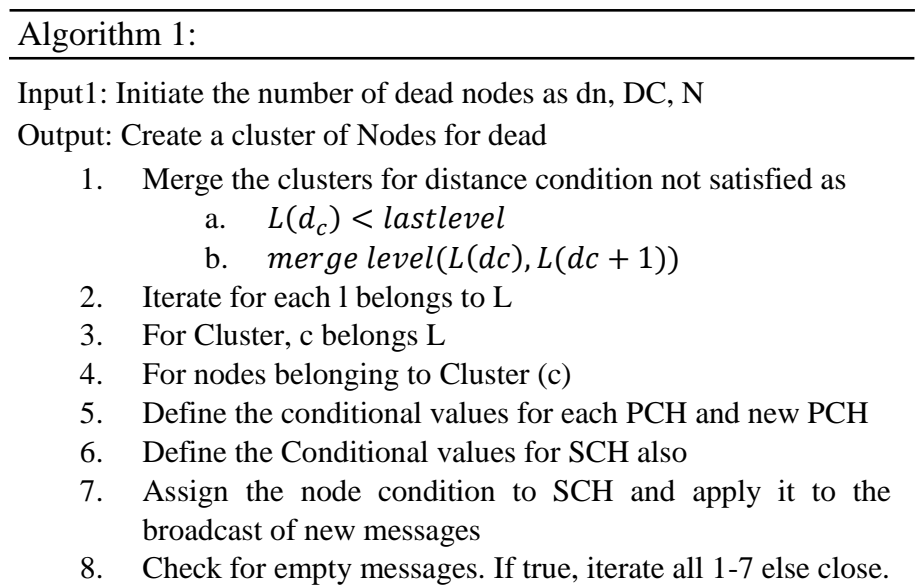

Algorithm 2: Intracluster routing algorithm

Input1: Initiate the number of nodes as $\mathrm{n}$

Output: Create a cluster of Nodes on $\mathrm{X}$ and $\mathrm{Y}$ directions which are $\mathrm{PCH}, \mathrm{SCH}$ and other affected nodes

1. Design messages for each set of Structures governing the Network as $\mathrm{S}$ and its distances from the sink.

2. Iterate the feature from all criteria $\mathrm{n}$ belongs to $\mathrm{L} 1$ for each node

3. Define minimum and maximum distances for the nodes to be operated

4. Assign a node for $\mathrm{PCH}$

5. Define the clusters for the same number of nodes in $\mathrm{S}$

6. Iterate for each set of clusters with $\mathrm{SCH}$

7. Finally, estimate the conditional values for Node distances and their cluster distances.

8. Iterate for all $\mathrm{S}$ belongs to $\mathrm{L}$

9. Broadcast the data with a message chosen with Cluster selected.

With features established based on the algorithm 1-2 this design effective implements congestion problem with routing methodology based on the structures (Fig-3 \& Fig-4). Now to improvise this design for other network problems such as Cluster-head energy, minimum energy, this design should be optimized with other routing scheme's apart from congestion scenarios where such concepts are mentioned in section -II.

\section{OPTIMIZING ALGORITHMS}

\section{A. Concept}

The purpose of the design might be as simple as lowering manufacturing costs or increasing production efficiency. An optimization algorithm is a method carried out repeatedly by comparing several solutions until an optimal or satisfying answer is discovered. Optimization became an element of computer-aided design operations with the introduction of computers. Today's optimization algorithms are divided into two categories:

1) Deterministic

2) Stochastic 


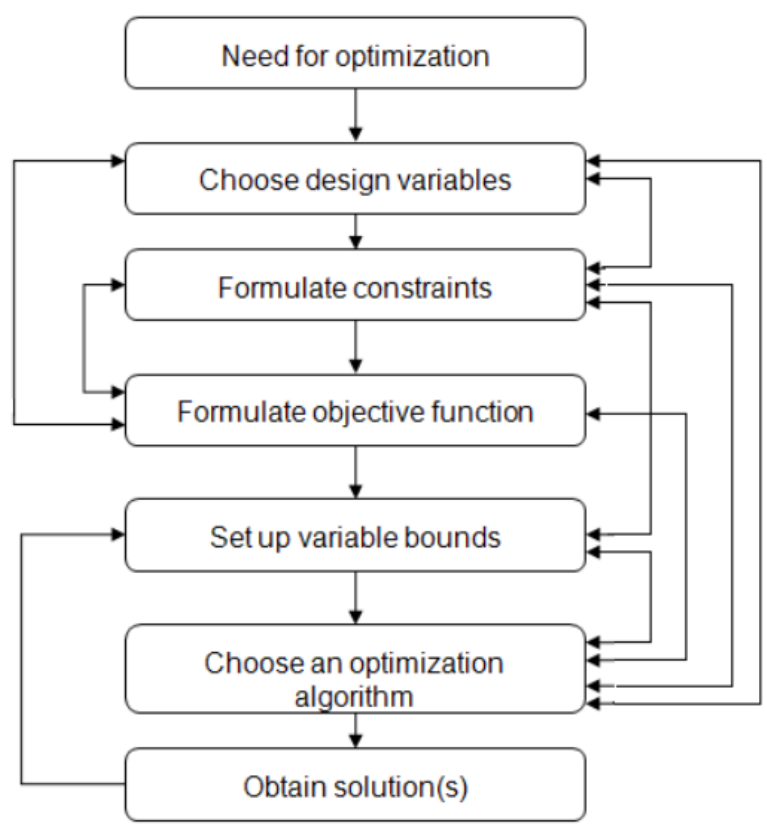

Fig. 5. Representing the Optimization Flow Diagram.

Our design improvises with the stochastic model on each Network parametric considered for optimizing. Since the optimization with energy-efficient is the prime scenario of the Node energy estimated and the node cluster energy modelled. In Fig 5 most specific feature is estimated with different variables and objective function with an optimization solution with solutions as fitness function values.

\section{B. Link State Optimization}

1) Initialize the design with a flag and graph where each node is utilized with maximum value.

2) Estimation on each tree graph is generated by selecting different MPR's based on Dijkstra's distance. We initiate the different scenario on the model where optimization is observed with minimum distance between each node estimated for selection of MPR's.

3) Optimizing the threshold values for each selected MPR's is generated.

4) Hopping on iterations changes would suffice the selection of MPR's selection onto the minimum energy model for given criteria.

Since, in section -I only congestion problem is illustrated with different routing structures on area implementation have been analyzed. While in section -II we improvise a conditional stochastic model with inequality criteria where this algorithm have been improvised to provide an effective solution for improved routing model with energy minimization problem as mention section -III and section -IV.

\section{PROPOSED MODEL}

\section{A. Concept}

Network parametric criteria with specific features of design modules as proposed with bitrate, Cluster-head energy, minimum energy and bandwidth usage. In this design, three features of the Network parametric have been introduced with the distance formulation as proposed with the hybrid routing protocol. The figure shows the optimum solution for all the parametric features with area modelling hybrid routing and, finally, energy minimization. These features are estimated with proposed algorithms SCI and HPTTA for energy inequality estimation for minimum energy solutions for each iteration.

This design features the stochastic model on the Network parametric to improve the performance factors for each iteration set. Fig 6 describes the importance of energy optimization with node and Routing have become the crucial aspect of the design for estimating different network parametric criteria. We propose a pervasive stochastic model to ensure the node and route optimal feature for every case of observed energy values. The Routing would improvise a distance feature model for each set of selected nodes from the OLS algorithm, and an improved distance approach with SCI inequality is applied for improved energy minimization.

\section{B. Area Set up for Topology Control}

In figure $7 \mathrm{a}$, we improvise a novel design scenario that effectively calculates the distances with R1, R2, R3 and R4 from the centre as $\mathrm{d} 1, \mathrm{~d} 2, \mathrm{~d} 3$ and $\mathrm{d} 4$. The adequate distances are formulated in section-3 for each region (1-4). The region in figure $7 \mathrm{a}$ with a star in-between represents the optimal regional transform for each tangential point as mentioned below:

\section{Let $p_{i}(i: 1$ to 4$)$ be the tangential points for star} region considered, where arc R1-R2 and arc R1-R4 will implicate the point P1 similarly for other arcs p2, p3, and p4 are represented.

\section{Let $E_{j}$ being the edges of the star connected to} circles with points $p_{i} i=1: 4$, here $\mathrm{j}$ varies from 1 to 10 . Even values of edge points are the critical points for which the tangential transform for the distances are applied.

$\sum_{i=1}^{N} \sqrt{\left(D_{x i}^{2}+D_{y i}^{2}\right) * \cos \left(\emptyset_{i}\right)}-\sqrt{\left(D_{x i}^{2}+D_{y i}^{2}\right) * \sin \left(\varnothing_{i}\right)}$

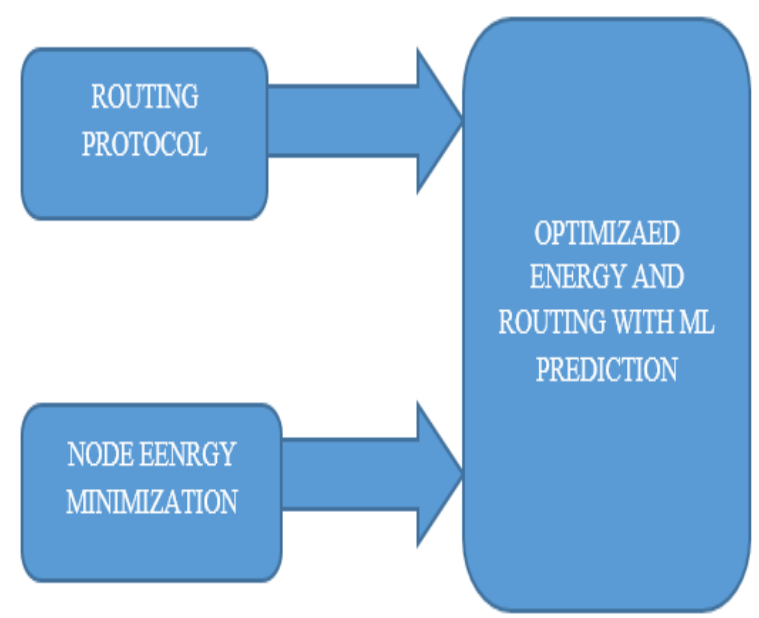

Fig. 6. Representing Optimization of Routing and Energy Minimization Block Diagram. 


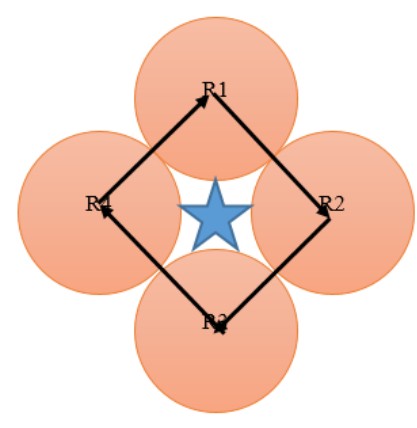

(a)

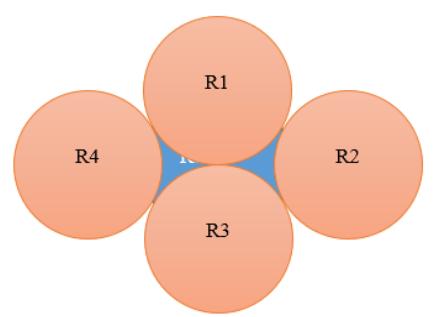

(b)

Fig. 7. (a) Representing the Pervasive Tangential Transform Model (b) Region Tangential with Projection Transform.

\section{Hybrid Routing Model}

With the Routing of hybrid feature, our design implicates the OLS algorithm as a proactive model and hybrid Manhattan distance feature for each OLS model, estimating the node minimum values for each node considered. The OLS algorithm improvises the design of MPR's multipoint relays, which are estimated with minimum values of distance based on the Hybrid Distance Formulations:

$$
\begin{aligned}
& D x 1=\sqrt{x_{i} \cos (45)}+\sqrt{y_{i} \sin (45)} \\
& D x 2=\sqrt{x_{i} \cos (15)}-\sqrt{y_{i} \sin (75)} \\
& D x=\min (D x 1, D x 2) \\
& \text { Final }_{\text {dist }}=\sqrt{D x 1^{2}+D x 2^{2}} \\
& \text { dist }_{x y}=\frac{x_{i}}{2 * \text { pi*final dist }}-y_{i} \sqrt{2 * \text { pi } * \text { Final dist }_{\text {din }}}
\end{aligned}
$$

\section{ENERGY-EFFICIENT FORMULATIONS}

\section{A. Node Energy Formulations}

We improvise an optimal solution for implicating Node estimations for hybrid routing as proposed, using formulations as mentioned for each set of nodes and selected clusters as:

This design for energy equation is measured with hybrid routing protocol as mentioned for topology Model:

$S(i)=\sum_{j=1}^{K} \sum_{i=1}^{N} \mu * F x(i, j)+\varphi * F z(i, j)$

The $\mu, \varphi$ The functionality of the $\mathrm{S}$ represents the design solution of which nodes appear at a given timing aspect where each set of the design parametric are considered with active and dead cells from the equation.

$$
\begin{aligned}
& F x(i>j)=\sum_{i=1}^{N}\left(n_{i} * D \min (i)+\sigma * S(i)\right) \\
& F z(i \leq j)=\sum_{i=1}^{N}\left(n_{i} * D_{\text {avg }}(i)\right)
\end{aligned}
$$

F represents the solution model for where all the active and alive nodes in cell regions are established with equation $1 . \mathrm{n} \_\mathrm{i}$ represents the number of active nodes and $\sigma$ being the best predicted based on ATGF for all iterations mentioned in the above algorithms.

$$
\begin{aligned}
& P E_{S C I}=F(i>k)+F(i \leq j) \\
& P E_{\text {Head_cluster }}=\gamma * W_{i} * D_{\text {min }}+\mu S C I(i)+E(i)
\end{aligned}
$$

Here $E(i)$ represents the entropy of each selected feature on node localization selected.

Hence the total Network energy estimated is:

$P E_{T}=P E_{\text {Head_cluster }}+P E_{S C I}$

Here $\gamma, \mu, \sigma$, estimated probabilities for the optimized values for the best solution are modified with the SCI algorithm probabilities for each random variable estimation. The overall estimated values implicate the least energy simulated parametric for iteration chosen.

\section{B. SCI Algorithm for Optimization of Minimum Energy}

Let there be two variables, $\mathrm{X}$ and $\mathrm{Y}$, the random probability for each node distance and energy optimization, on selecting optimal distance search and link-state protocol as mentioned in section 4.

$P\left(\frac{X}{D_{\text {mini }}}\right)=P(X) P\left(X \cap D_{\text {mini }}\right) / P\left(D_{\text {mini }}\right)$

$P\left(\frac{Y}{E_{\text {mini }}}\right)=P(Y) P\left(Y \cap E_{\text {mini }}\right) / P\left(E_{\text {mini }}\right)$

Since the conditionality of the stochastic model, $P\left(E_{\text {mini }} /\right.$ $\left.D_{\text {mini }}\right)$ is a conditional value for each Random variable $\mathrm{X}$ and $\mathrm{Y}$ for distance and energy minimization.

$P\left(\frac{E_{\text {mini }}}{D_{\text {mini }}}\right)=P\left(E_{\text {mini }}\right) * \frac{P\left(E_{\text {mini }} \cap D_{\text {mini }}\right)}{P\left(D_{\text {mini }}\right)}$

For $X \in D_{\text {mini }}$ and $Y \in E_{\text {mini }}$ hence,

$P(E \cap D)=P(E)+P(X \cap D)+P\left(\frac{X}{D_{\text {mini }}}\right) * \frac{P(D)}{P(X)}+$

$P\left(D_{\text {mini }}\right)+P\left(\frac{Y}{E_{\text {mini }}}\right) * \frac{P(E)}{P(Y)}+P(Y \cap E)$

Hence $P(E)_{\min } \approx P\left(\frac{X}{D_{\text {mini }}}\right) * \frac{P(D)}{P(X)}+P\left(D_{\text {mini }}\right)+P\left(\frac{Y}{E_{\text {mini }}}\right) *$ $\frac{P(E)}{P(Y)}$

\section{RESULTS AND DISCUSSION}

\section{A. Output Simulations}

In this design, we have implicated the different features of the Network with different nodes and its multipoint routes estimated from the hybrid routing area transform model. The simulations are estimated with these nodes, and an optimal solution for the energy minimization and 1.8-1.9 bit rate is observed for the two algorithms proposed as SCI and HTTA from equation 3 . 
In figure 8 , energy values are plotted with the minimum number of nodes in one direction. The minimum values for each set of iterations are observed with $-220 \mathrm{~dB}$ for $50 \mathrm{X} 50$ net, $-238 \mathrm{~dB}$ for $100 \mathrm{X} 100$ and $-220 \mathrm{~dB}$ for $150 \mathrm{X} 150$. Formulations from 1-19 are implemented for each setup iteration and compared with minimum energy values, bit rate and finally, the error values for each random factor chosen for optimizing the data as mentioned in figure 9-11.

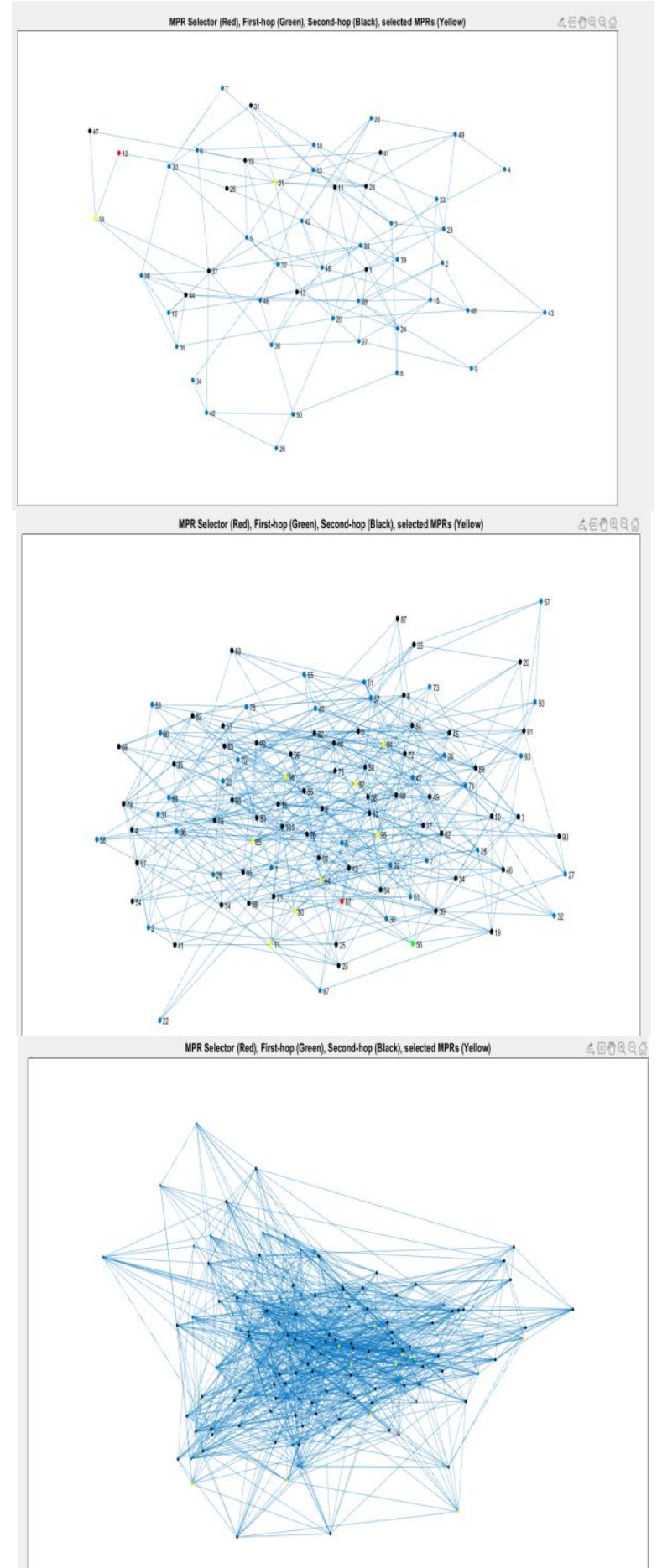

Fig. 8. Representing the WSN Structure for (a) $50 X 50$ Nodes, (b) $100 \times 100$ Nodes (c) 150x150 Nodes.

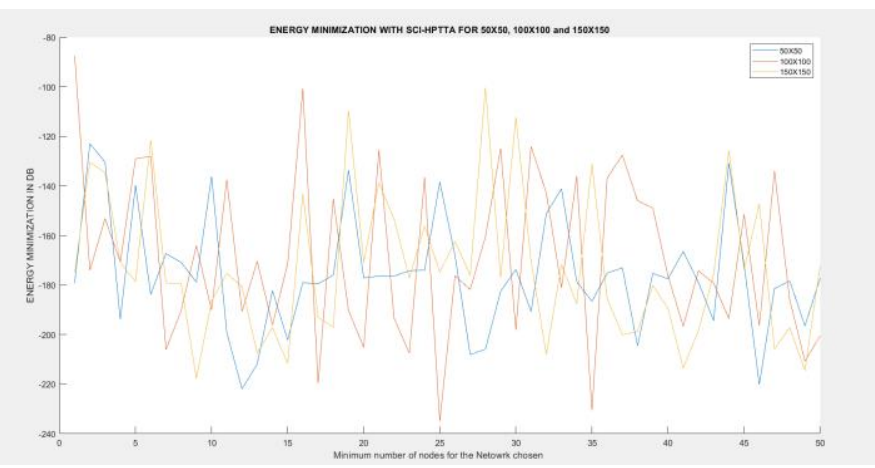

Fig. 9. Representing the Energy Values for (a) 50X50, (b) 100X100, (c) $150 \times 150$.

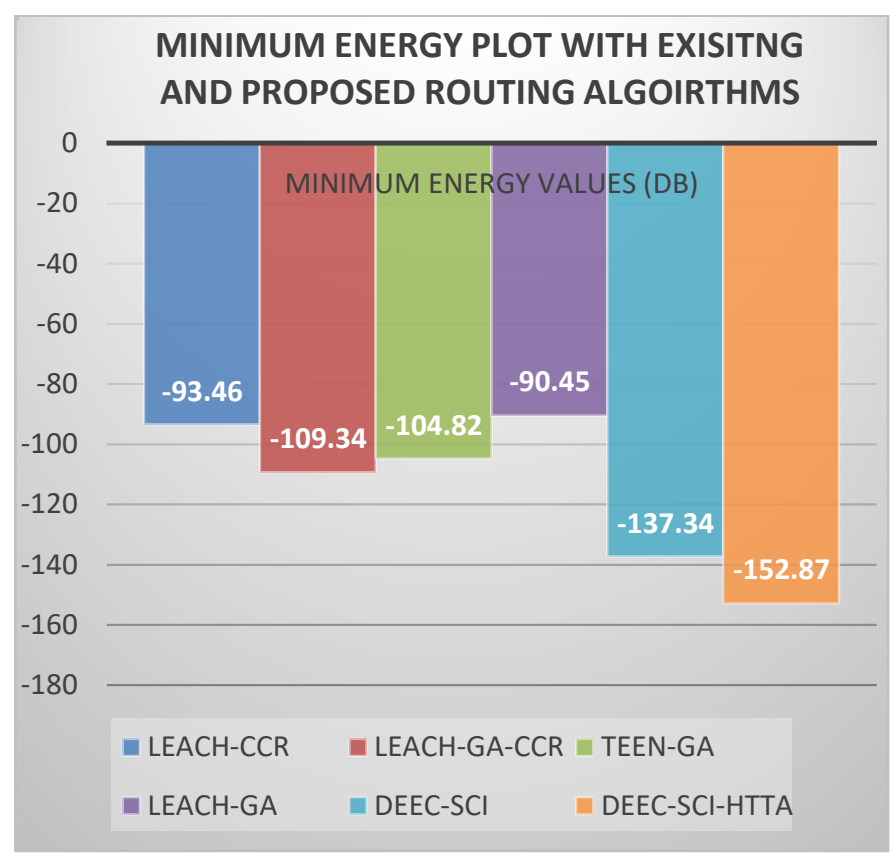

Fig. 10. Representing the Minimum Energy Values for Existing and Proposed Algorithms.

The minimum energy values of each algorithm mention with LEACH $\{\mathrm{CCR}, \mathrm{GA}, \mathrm{GA}-\mathrm{CCR}\}, \mathrm{DEEC}\{\mathrm{SCI}, \mathrm{SCI}-$ HTTA proposed algorithm\}, TEEN-GA are estimated with references [21,22]. The DEEC-SCI and DEEC-SCI-HTTA algorithm have been improvised on different solutions with each estimate on the equations from (15) and (18).

In Fig-11 and Fig-12, our design depicts with overall bit rate estimation for each transmitted data from the sender to the destination. To increase the bit of the transmission our design utilizes hybrid routing protocol with as proposed with area estimation structure. These area estimation analyses the correct distance from each node and its transmitter data to the send via SCI algorithm. Finally, the error optimization with hybrid routing scheme is analysed and implicated on the design feature. The error values are implicated with optimization algorithm as Stochastic model with SCI algorithm. 


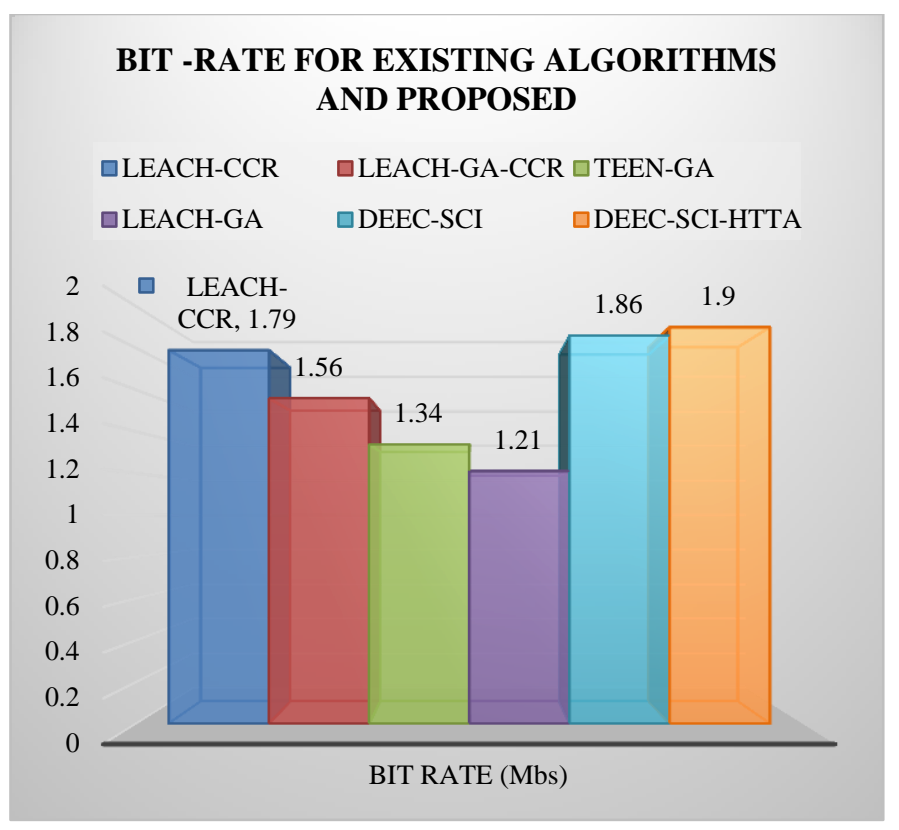

Fig. 11. Representing the Bit Rate Values for Existing and Proposed Algorithms.

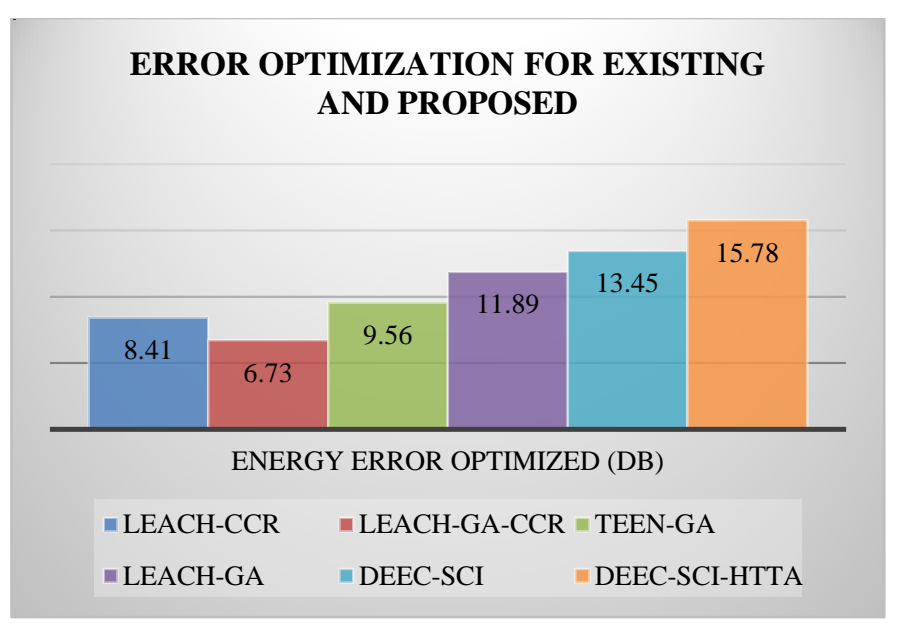

Fig. 12. Representing the Error Values in $\mathrm{dB}$ for Existing and Proposed Algorithms.

\section{B. Tabulations}

The Table 1a) implicates on different LEACH protocols from Existing model CCR referring to Congestion control route. The Fig-3 and Fig-4are the prime features for provision of routing with and without congestion for set of nodes as energy minimization. GA is other optimization algorithm considered for route minimization and its distance optimizing on each iteration for every node considered. Hence, Algorithm-2 from Table 1a) provisions best outcome for energy, bitrate and error in DB while others are less. So, in table-1b) is implemented based on the proposed algorithms as mention in section-III and IV encapsulating the different propositions of the energy models and its analysis for distance routes and node clusters minimizations. This results overall improved feature more than $12 \%$ on each of the parameter considered.
TABLE I. REPRESENTS THE DIFFERENT ALGORITHMS IMPLICATED WITH LEACH AND TEEN

\begin{tabular}{|l|l|l|l|l|}
\hline \multirow{2}{*}{ SNO } & \multicolumn{4}{|l|}{ Comparison table } \\
\cline { 2 - 5 } & Existing Algorithms & Min-Energy & Bitrate & Error in dB \\
\hline 1 & LEACH-CCR & -93.46 & 1.79 & 8.41 \\
\hline 2 & LEACH-GA-CCR & -109.34 & 1.56 & 6.73 \\
\hline 3 & TEEN-GA & -104.82 & 1.34 & 9.56 \\
\hline 4 & LEACH-GA & -90.45 & 1.21 & 11.89 \\
\hline
\end{tabular}

(B) REPRESENTS THE PROPOSED ALGORITHMS DEEC-SCI AND SCI-HTTA

\begin{tabular}{|l|l|l|l|l|}
\hline \multirow{2}{*}{ SNO } & \multicolumn{4}{|l|}{ Comparison table } \\
\cline { 2 - 5 } & Proposed Algorithms & Min-Energy & Bitrate & Error in dB \\
\hline 1 & DEEC-SCI & -137.34 & 1.86 & 13.45 \\
\hline 2 & DEEC-SCI-HTTA & -152.87 & 1.9 & 15.78 \\
\hline
\end{tabular}

\section{VII.CONCLUSION}

In WSN applications, power consumption is a common difficulty regardless of the task. To maintain the Network's overall efficiency, this design model with SCI and HTTA is implemented with energy minimization is required. This work intended with Routing and its area-based design model with tangential transform for different Network parametric as SCIHTTP model with DEEC is proposed. The optimized features on performance parametric suggest an outstanding improvement on minimum energy values for the proposed two algorithms. In figure 2, we have implicated the DEEC-SCI and DEEC -SCI-HTTA features for the proposed model. These few algorithms are being considered with the proposed improvement feature on Routing speed characteristics. From section -6 , all tabulations and plotting features are represented via Matlab-2019b. The practical value minimum value for each DEEC-SCI-HTTA is observed in terms of -200 to -300 $\mathrm{dB}$ for a single iteration.

SCOPE:

1) To estimate heterogenous network for optimizing coverage problem

2) Improvise a Novel scheme on optimal energy nodes and its provision on hardware model for WSN network.

\section{REFERENCES}

[1] K Abdul Basith, T.N. Shankar, "Hybrid state analysis with improved firefly optimized linear congestion models of WSNs for DDOS \& CRA attacks", URL link: Hybrid state analysis with improved firefly optimized linear congestion models of WSNs for DDOS \& CRA attacks [PeerJ], pp-11-14,DOP:2022-01-27.

[2] Muhammad Atif Ur Rehman; Rehmat Ullah; Byung-Seo Kim; Boubakr Nour; Spyridon Mastorakis, "CCIC-WSN: An Architecture for SingleChannel Cluster-Based Information-Centric Wireless Sensor Networks", IEEE Internet of Things Journal (Volume: 8, Issue: 9, May 1, 12021) Page(s): 7661 - 7675 Electronic ISSN: 2327-4662.

[3] F. Fernando Jurado-Lasso; Ken Clarke; Ampalavanapillai Nirmalathas, "Performance Analysis of Software-Defined Multihop Wireless Sensor Networks", IEEE Systems Journal ( Volume: 14, Issue: 4, Dec. 2020).

[4] T. M. Behera, S. K. Mohapatra, U. C. Samal, M. S. Khan, M. Daneshmand and A. H. Gandomi, "Residual energy-based cluster-head selection in WSNs for IoT application", IEEE Internet Things J., vol. 6, no. 3, pp. 5132-5139, Jun. 2019. 
[5] M. Ndiaye, G. P. Hancke and A. M. Abu-Mahfouz, "Software-defined networking for improved wireless sensor network management: A survey", Sensors, vol. 17, no. 5:1031, pp. 1-32, 2017.

[6] O. Salem, A. Serhrouchni, A. Mehaoua and R. Boutaba, "Event detection in wireless body area networks using Kalman filter and power divergence", IEEE Trans. Netw. Service Manag., vol. 15, no. 3, pp. 1018-1034, Sep. 2018.

[7] C. Chen, J. Yan, N. Lu, Y. Wang, X. Yang and X. Guan, "Ubiquitous monitoring for industrial cyber-physical systems over relay-assisted wireless sensor networks", IEEE Trans. Emerg. Topics Comput., vol. 3, no. 3, pp. 352-362, Sep. 2015.

[8] S. Misra, S. Bera, M. Achuthananda, S. K. Pal and M. S. Obaidat, "Situation-aware protocol switching in software-defined wireless sensor network systems", IEEE Syst. J., vol. 12, no. 3, pp. 2353-2360, Sep. 2018.

[9] M. Ndiaye, G. P. Hancke and A. M. Abu-Mahfouz, "Software-defined networking for improved wireless sensor network management: A survey", Sensors, vol. 17, no. 5:1031, pp. 1-32, 2017.

[10] K. Latif, N. Javaid, M. N. Saqib, Z. A. Khan and N. Alrajeh, "Energy consumption model for density controlled divide-and-rule scheme for energy-efficient routing in wireless sensor networks", Int. J. Ad Hoc Ubiquitous Comput., vol. 21, no. 2, pp. 130-139, 2016.

[11] G. Ateniese et al., "Low-cost standard signatures for energy-harvesting wireless sensor networks", ACM Trans. Embedded Comput. Syst., vol. 16, no. 3, 2017.

[12] F. Wu, L. Xu, S. Kumari and X. Li, "A privacy-preserving and provable user authentication scheme for wireless sensor networks based on internet of things security", J. Ambient Intell. Humanized Comput., vol. 8, no. 1, pp. 101-116, 2017.

[13] H. Zhang and Z. Pan, "Cross-voting SVM method for multiple vehicle classification in wireless sensor networks", Sensors, vol. 18, no. 9, pp. 3108, 2018.

[14] N. Jain, S. Verma and M. Kumar, "Adaptive locally linear embedding for node localization in sensor networks", IEEE Sensors J., vol. 17, no. 9, pp. 2949-2956, May 2017.

[15] A. Singh and S. Verma, "Graph Laplacian regularization with Procrustes analysis for sensor node localization", IEEE Sensors J., vol. 17, no. 16, pp. 5367-5376, Aug. 2017.

[16] W. Heinzelman, A. P. Chandrakasan, H. Balakrishnan, and A. C. Smith, "Application-specific protocol architectures for wireless networks," PhD dissertation, Massachusetts Inst. Technol., Cambridge, MA, USA, 2000.

[17] V. Loscri, G. Morabito, and S. Marano, "A two-levels hierarchy for lowenergy adaptive clustering hierarchy (TL-LEACH)," in Proc. IEEE Veh. Technol. Conf., Sep. 2005, vol. 62, no. 3, pp. 1809-1813.

[18] L. Lijun, W. Hongtao, and C. Peng, "Discuss in round rotation policy of hierarchical route in wireless sensor networks," Proc. Int. Conf. Wireless Commun., Netw. Mobile Comput., Sep. 2006, pp. 1-5.
[19] F. Xiangning and S. Yulin, "Improvement on LEACH protocol of wireless sensor network," in Proc. Int. Conf. Sensor Technol. Appl., Oct. 2007, pp. 260-264.

[20] H. Junping, J. Yuhui, and D. Liang, "A time-based cluster-head selection algorithm for LEACH," in Proc. IEEE Symp. Comput. Commun., Jul. 2008, pp. 1172-1176.

[21] M. S. Ali, T. Dey, and R. Biswas, "ALEACH: Advanced LEACH routing protocol for wireless microsensor networks," in Proc. Int. Conf. Elect. Comput. Eng., Dec. 2008, pp. 909-914.

[22] W. Wang, Q. Wang, W. Luo, M. Sheng, W. Wu, and L. Hao, "LEACHH: An improved routing protocol for collaborative sensing networks," in Proc. Int. Conf. Wireless Commun. Signal Process., Nov. 2009, pp. 1-5.

[23] G. Yi, S. Guiling, L. Weixiang, and P. Yong, "Recluster-LEACH: A recluster control algorithm based on density for wireless sensor network," in Proc. 2nd Int. Conf. Power Electron. Intell. Transp. Syst. (PEITS), vol. 3, Dec. 2009, pp. 198-202.

[24] A. Yektaparast, F.-H. Nabavi, and A. Sarmast, "An improvement on LEACH protocol (Cell-LEACH)," in Proc. 14th Int. Conf. Adv. Commun. Technol. (ICACT), Feb. 2012, pp. 992-996.

[25] G. N. Basavaraj and C. D. Jaidhar, "H-LEACH protocol with modified cluster head selection for WSN," in Proc. Int. Conf. Smart Technol. Smart Nation, Aug. 2017, pp. 30-33.

[26] A. Razaque, M. Abdulgader, C. Joshi, F. Amsaad, and M. Chauhan, "PLEACH: Energy-efficient routing protocol for wireless sensor networks," in Proc. IEEE Long Island Syst., Appl. Technol. Conf. (LISAT), Apr. 2016, pp. 1-5.

[27] Uma Maheswari Durairaj; Sudha Selvaraj, "Two-Level Clustering and Routing Algorithms to Prolong the Lifetime of Wind Farm-Based WSN", IEEE Sensors Journal ( Volume: 21, Issue: 1, Jan.1, 1 2021), DOI: 10.1109/JSEN.2020.3015734.

[28] Tarunpreet Kaur; Dilip Kumar, "MACO-QCR: Multi-Objective ACOBased QoS-Aware Cross-Layer Routing Protocols in WSN", IEEE Sensors Journal ( Volume: 21, Issue: 5, March1, 1 2021), Electronic ISSN: 1558-1748.

[29] Xiuwen Fu; Yongsheng Yang; Octavian Postolache, "Sustainable Multipath Routing Protocol for Multi-Sink Wireless Sensor Networks in Harsh Environments", IEEE Transactions on Sustainable Computing ( Volume: 6, Issue: 1, Jan.-March 1 2021), Electronic ISSN: 2377-3782.

[30] Shaha Al-Otaibi, Amal Al-Rasheed,Romany F. Mansour, Gyanendra Prasad Joshi, Woong Cho, " Hybridization of Metaheuristic Algorithm for Dynamic Cluster-Based Routing Protocol in Wireless Sensor Networksx", IEEE Access ( Volume: 9), Date of Publication: 08 June 2021. 